However, there is a lot of health and healthcare problems caused by the nuclear accident, which is not owing to radiation. Objectives So far little incentive has been made on gaining holistic view of emerging health problems after the nuclear accident. Our aim is to share quantitative data about these issues and to appeal for the need of systematic approach to global assessment of health impact by the nuclear accident.

Methods Experience data was collected from the experience of medical doctors and firefighters working at on-site hospital. Health data of the residents wer collected by the Soma City local government.

Result After the evacuation order by the national government, those who did not have access to the information, such as the elderlies living alone, were left within the evacuation zone. This is shown by the fact that within a week after the evacuation order, 7 emergency calls were made from within the evacuation zone. Another case is that long-term evacuation to temporary housings deteriorate health of the elderly. Health check-ups conducted by Soma City local government revealed increased risks of muscle weakness, obesity and diabetes are apparent 1 year after the disaster. Not only health problems among the residents, those of decontamination workers are increasingly a burden of health facilities in Fukushima. Being seasonal workers under precarious employment, prevalence rates of pre-existing chronic conditions such as hypertension and diabetes are high among these workers. As a result, healthcare for these workers are now an increasing burden on the frontline health facilities. Conclusion To establish effective DRR for a nuclear accident, holistic view on health is required.

\title{
117 WHAT IS HEALTH IMPACT CAUSED BY A NUCLEAR ACCIDENT?
}

Sae Ochi. Internal Medicine, Soma Central Hospital, Soma, Japan

10.1136/bmjopen-2015-forum2015abstracts.117

Background After the Fukushima Daiichi nuclear power plant accident in 2011, a considerable attention has been paid on radiation exposure level among the residents in Fukushima. 